\title{
Optimal Inequality in the One-Parameter Arithmetic and Harmonic Means
}

\section{Mohammed El Mokhtar Ould El Mokhtar'1, Hamad Alharbi²}

${ }^{1}$ Qassim University, Qassim, KSA

${ }^{2}$ Shaqra University, Riyadh, KSA

Email: med.mokhtar66@yahoo.fr, M.labdi@qu.edu.sa,halharby@su.edu.sa

How to cite this paper: El Mokhtar Ould El Mokhtar, M. and Alharbi, H. (2020) Optimal Inequality in the One-Parameter Arithmetic and Harmonic Means. Open Access Library Journal, 7: e6586.

https://doi.org/10.4236/oalib.1106586

Received: July 6, 2020

Accepted: July 21, 2020

Published: July 24, 2020

Copyright $\odot 2020$ by author(s) and Open Access Library Inc.

This work is licensed under the Creative Commons Attribution International License (CC BY 4.0).

http://creativecommons.org/licenses/by/4.0/

\begin{abstract}
This research work considers the inequalities: (Ieq). The researchers attempt to find an answer as to what are the best possible parameters $\alpha, \beta$ that (Ieq) can be hold? The main tool is the optimization of some suitable functions that we seek to find out.
\end{abstract}

\section{Subject Areas}

Mathematical Analysis

\section{Keywords}

One-Parameter, Arithmetic, Harmonic Means

\section{Introduction}

In this paper we consider the following inequalities:

$$
\alpha A(a, b)+(1-\alpha) H(a, b) \leq J_{p}(a, b) \leq \beta A(a, b)+(1-\beta) H(a, b) \quad(\text { Inq) (1) }
$$

with $A(a, b)=\frac{a+b}{2} ; H(a, b)=\frac{2 a b}{a+b}$

$$
J_{p}(a, b)= \begin{cases}\frac{p\left(a^{P+1}-b^{P+1}\right)}{(P+1)\left(a^{P}-b^{P}\right)} ; & a \neq b ; \quad P \neq 0,-1 \\ \frac{a-b}{\ln a-\ln b}, & a \neq b ; \quad P=0 \\ \frac{a b(\ln a-\ln b)}{a-b}, & a \neq b ; \quad P=-1 \\ a, \quad & a=b\end{cases}
$$

Our motivation of this study is to find out such inequality that arises in the 
search for determination of a point of reference about which some function of variants would be minimum or maximum. Since very early times, people have been interested in the problem of choosing the best single quantity, which could summarize the whole information contained in a number of observations (measurements). Moreover, the theory of means has its roots in the work of the Pythagorean who introduced the harmonic, geometric, and arithmetic means. Peter et al. [1] introduced seven other means and gave the well-known elegant geometric proof of the celebrated inequalities among the harmonic, geometric, and arithmetic means. The strong relations and introduction of the theory of means with the theories of inequalities, function equations, probability and statistics add greatly to its importance. This single element is usually called a means or averages. The term "means" or "average" (middle value) has for a long time been used in all branches of human activity. The main objective of this research work is to present optimization of inequality in the one-parameter, arithmetic and harmonic means.

The basic function of mean value is to represent a given set of many values by some single value. In [2], the author was the first time introduced power means defined the meaning of the term "representation" as determination of appoint of reference about which some function of variants would be minimum. More recently the means were the subject of research and study whereas essential areas in several applications such as: physics, economics, electrostatics, heat conduction, medicine and even in meteorology. It can be observed that the power mean $M_{p}(a, b)$ of order $p$ can be rewritten as (see as [3])

$$
M_{p}(a, b)= \begin{cases}\left(\frac{a^{p}+b^{p}}{2}\right)^{\frac{1}{p}} ; & p \neq 0 \\ \sqrt{a b} ; & p=0\end{cases}
$$

If we denote by

$$
A(a, b)=\frac{1}{2}(a+b), G(a, b)=\sqrt{a b} \text { and } H(a, b)=\frac{2 a b}{a+b},
$$

the arithmetic means, geometric means and harmonic means of two positive numbers $a$ and $b$, respectively. In addition, the logarithmic and identric means of two positive real numbers $a$ and $b$ defined by [4]

$$
\begin{aligned}
& L(a, b)= \begin{cases}\frac{b-a}{\log b-\log a} & a \neq b \\
a & a=b\end{cases} \\
& I(a, b)= \begin{cases}\frac{1}{\mathrm{e}}\left(\frac{b^{b}}{a^{a}}\right)^{11(b-a)} & a \neq b \\
a & a=b\end{cases}
\end{aligned}
$$

Several authors investigated and developed a relationship of optimal inequalities between the various means.

The well-known inequality that: 


$$
\begin{aligned}
\min \{a, b\} & \leq H(a, b)=M_{-1}(a, b) \leq G(a, b)=M_{0}(a, b) \\
& \leq L(a, b) \leq I(a, b) \leq A(a, b)=M_{1}(a, b) \leq \max \{a, b\}
\end{aligned}
$$

and all inequalities are strict for $a \neq b$.

In [4], researchers studied what are the best possible parameters $\alpha_{1}, \alpha_{2}, \beta_{1}$ and $\beta_{2}$ by two theorems:

Theorem (1) the double inequality: -

$$
\alpha_{1} A(a, b)+\left(1-\alpha_{1}\right) H(a, b) \leq L(a, b) \leq \beta_{1} A(a, b)+\left(1-\beta_{1}\right) H(a, b)
$$

holds for all $a, b>0$ if and only if $\alpha_{1} \leq 0$ and $\beta_{1} \geq \frac{2}{3}$ when proved that the parameters $\alpha_{1} \leq 0$ and $\beta_{1} \geq \frac{2}{3}$ cannot be improved.

Theorem (2) the double inequality: -

$$
\alpha_{2} A(a, b)+\left(1-\alpha_{2}\right) H(a, b) \leq L(a, b) \leq \beta_{2} A(a, b)+\left(1-\beta_{2}\right) H(a, b)
$$

holds for all $a, b>0$ if and only if $\alpha_{2} \leq \frac{2}{\mathrm{e}}$ and $\beta_{2} \geq \frac{5}{6}$ when proved that the parameters $\alpha_{2} \leq \frac{2}{\mathrm{e}}$ and $\beta_{2} \geq \frac{5}{6}$ cannot be improved.

Interestingly in [1] B. Long et al., proved that the following results: $M_{0}(a, b)$ and $M_{t l 3}(a, b)$ are the best possible lower and upper power bounds for the generalized logarithmic mean $L_{t}(a, b)$ for any fixed $t>0$ the double inequalities

$$
M_{0}(a, b)<L_{t}(a, b)<M_{t l 3}(a, b)
$$

holds for all $a, b>0$ with $a \neq b$, and they found $L_{2}(a, b)$ the optimal lower generalized logarithmic means bound for the identric means $I(a, b)$ for inequalities $L_{2}(a, b)<I(a, b)$ holds for all $a, b$ are positive numbers with $a \neq b$. Pursuing another line of investigation, in [5] the authors showed the sharp upper and lower bounds for the Neuman-sandor NS $(a, b)$ [6] in terms of the liner convex combination of the logarithmic means $L(a, b)$ and second seiffert means $T(a, b)$ [7] of two positive numbers $a$ and $b$, respectively for the double inequalities

$$
\alpha L(a, b)+(1-\alpha) T(a, b) \leq N S(a, b) \leq \beta L(a, b)+(1-\beta) T(a, b)
$$

holds for all $a, b>0$ with $a \neq b$ is true if and only if $\alpha \geq \frac{1}{4}$ and $\beta \leq 1-\pi l[4 \log (1+\sqrt{2})]$.

In [8] have improvements and refinements by H.Z. Xu et al., for they found several sharp upper and lower bounds for the Sandor-yang means $R_{\mathrm{QA}}(a, b)$ and $R_{A Q}(a, b)$ [9] [10] in terms of combinations of the arithmetic means $A(a, b)$ and the contra-harmonic mean $C(a, b)$ [11] [12].

The authors have to proven our main results several lemmas find the best possible parameters $\alpha_{i}, \beta_{i} \in(i=1,2,3,4)$ such that the double inequalities 


$$
\begin{gathered}
c^{\alpha_{1}}(a, b) A^{1-\alpha_{1}}(a, b)<R_{Q A}(a, b)<c^{\beta_{1}}(a, b) A^{1-\beta_{1}}(a, b) \\
c^{\alpha_{2}}(a, b) A^{1-\alpha_{2}}(a, b)<R_{Q A}(a, b)<c^{\beta_{2}}(a, b) A^{1-\beta_{2}}(a, b) \\
\alpha_{3}\left[\frac{1}{3} C(a, b)+\frac{2}{3} A(a, b)\right]+\left(1-\alpha_{3}\right) C^{113}(a, b) A^{2 l 3}(a, b) \\
<R_{Q A}(a, b)<\beta_{3}\left[\frac{1}{3} C(a, b)+\frac{2}{3} A(a, b)\right]+\left(1-\beta_{3}\right) C^{113}(a, b) A^{2 l 3}(a, b) \\
\alpha_{4}\left[\frac{1}{6} C(a, b)+\frac{5}{6} A(a, b)\right]+\left(1-\alpha_{4}\right) C^{116}(a, b) A^{5 l 6}(a, b) \\
<R_{A Q}(a, b)<\beta_{4}\left[\frac{1}{6} C(a, b)+\frac{5}{6} A(a, b)\right]+\left(1-\beta_{4}\right) C^{116}(a, b) A^{5 l 6}(a, b)
\end{gathered}
$$

holds for all $a, b>0$ with $a \neq b$.

\section{Main Results}

Our main results are set in the following theorem:

\section{Theorem 1}

1) Assume $a>0, b>0$ with $\frac{a}{b}>1$ then,

a) if $p \in\left(-1, p_{1}\right)$ where $p_{1}=\frac{-9+\sqrt{73}}{2}<0$. There exist $\alpha_{*}$ and $\alpha^{*}$ reals such that, if $\alpha_{*}<\alpha<\alpha^{*}<\beta$ then the double inequality (Inq) holds.

b) if $p=0$. If $\alpha<0$ and $\frac{3}{2}<\beta<2$ then the double inequality (Inq) holds.

c) if $p=-1$. If $\alpha<0$ and $\beta>\frac{1}{3}$ then the double inequality (Inq) holds.

2) If $a=b$ then then the double inequality (Inq) holds for all $\alpha$ and $\beta$ reals.

Proof. 1) Assuming $a>0, b>0$ with $\frac{a}{b}>1$

First case a): we have

$$
\begin{aligned}
\alpha\left(\frac{a+b}{2}\right)+(1-\alpha)\left(\frac{2 a b}{a+b}\right) & \leq \frac{p\left(a^{p+1}-b^{p+1}\right)}{(p+1)\left(a^{p}-b^{p}\right)} \leq \beta\left(\frac{a+b}{2}\right)+(1-\beta)\left(\frac{2 a b}{a+b}\right) \\
a & \neq b ; \quad p \neq 0,-1 ; \quad a>b .
\end{aligned}
$$

Set $t=\frac{a}{b}>1$. Then, we obtain

$$
\alpha\left(\frac{b(t+1)}{2}\right)+(1-\alpha)\left(\frac{2 t b}{t+1}\right) \leq \frac{p b\left(t^{p+1}-1\right)}{(p+1)\left(t^{p}-1\right)} \leq \beta\left(\frac{b(t+1)}{2}\right)+(1-\beta)\left(\frac{2 t b}{t+1}\right)
$$

We start by showing that

$$
\alpha\left(\frac{b(t+1)}{2}\right)+(1-\alpha)\left(\frac{2 t b}{t+1}\right)-\frac{p b\left(t^{p+1}-1\right)}{(p+1)\left(t^{p}-1\right)} \leq 0
$$




$$
\begin{aligned}
\Leftrightarrow & \frac{\alpha b(t+1)^{2}(p+1)\left(t^{p}-1\right)+4(1-\alpha) t b(p+1)\left(t^{p}-1\right)}{2(t+1)(p+1)\left(t^{p}-1\right)} \\
& +\frac{-2(t+1) p b\left(t^{p+1}-1\right)}{2(t+1)(p+1)\left(t^{p}-1\right)} \leq 0
\end{aligned}
$$

Because $p>-1$, we have $2(t+1)(p+1)\left(t^{p}-1\right)>0$ therefore the study amounts to proving that

$$
\begin{aligned}
& \alpha b(t+1)^{2}(p+1)\left(t^{p}-1\right)+4(1-\alpha) t b(p+1)\left(t^{p}-1\right) \\
& -2(t+1) p b\left(t^{p+1}-1\right) \leq 0 .
\end{aligned}
$$

Let

$$
\begin{aligned}
f(t)= & \alpha b(t+1)^{2}(p+1)\left(t^{p}-1\right)+4(1-\alpha) t b(p+1)\left(t^{p}-1\right) \\
& -2(t+1) p b\left(t^{p+1}-1\right)
\end{aligned}
$$

We have to prove that the function $f$ is negative under certain conditions on the parameters $\alpha, \beta$ and $p$, a.e: $f(t) \leq 0$. So

$$
\begin{aligned}
f(t)= & \alpha b(t+1)^{2}(p+1)\left(t^{p}-1\right)+4(1-\alpha) t b(p+1)\left(t^{p}-1\right) \\
& -2(t+1) p b\left(t^{p+1}-1\right) \leq 0
\end{aligned}
$$

Because $f(1)=0$, it will suffice to show that $f$ is decreasing for all $t>1$. Which amounts to studying the sign of the derivative $f^{\prime}$ of $f$. We have:

$$
\begin{aligned}
f^{\prime}(t)= & {[\alpha b(p+1)(p+2)-2 b p(p+2)] t^{p+1} } \\
& +\left[2 \alpha b(p+1)^{2}+4(1-\alpha) b(p+1)^{2}-2 b p(p+1)\right] t^{p} \\
& +[\alpha b p(p+1)] t^{p-1}+[-2 \alpha b(p+1)] t \\
& +[-2 \alpha b(p+1)-4(1-\alpha) b(p+1)+2 b p]
\end{aligned}
$$

Because $f^{\prime}(1)=0$, it will suffice to show that $f^{\prime}$ is decreasing for all $t>1$. Which amounts to studying the sign of the derivative $f$ of $f^{\prime}$. We have:

$$
\begin{aligned}
f^{\prime \prime}(t)= & {\left[\alpha b(p+1)^{2}(p+2)-2 b p(p+2)(p+1)\right] t^{p} } \\
& +\left[2 \alpha b p(p+1)^{2}+4(1-\alpha) b p(p+1)^{2}-2 b p^{2}(p+1)\right] t^{p-1} \\
& +[\alpha b p(p+1)(p-1)] t^{p-2}+[-2 \alpha b(p+1)]
\end{aligned}
$$

Likewise we find that $f^{\prime \prime}(1)=0$ so it will suffice to show that $f^{\prime \prime}$ is decreasing for all $t>1$. Which amounts to studying the sign of the derivative $f^{\prime \prime \prime}$ of $f^{\prime \prime}$. We have:

$$
\begin{aligned}
f^{\prime \prime \prime}(t)= & {\left[\alpha b p(p+1)^{2}(p+2)-2 b p^{2}(p+2)(p+1)\right] t^{p-1} } \\
& +\left[2 \alpha b p(p+1)^{2}(p-1)+4(1-\alpha) b p(p+1)^{2}(p-1)\right. \\
& \left.-2 b p^{2}(p+1)(p-1)\right] t^{p-2}+[\alpha b p(p+1)(p-1)(p-2)] t^{p-3}
\end{aligned}
$$

and we get 


$$
f^{\prime \prime \prime}(1)=6 \alpha b p(p+1)-2 p b(p+1)[p+2]
$$

Since $p \in\left(-1, p_{1}\right)$ where $p_{1}=\frac{-9+\sqrt{73}}{2}<0$ so, we will have the following equivalence

$$
f^{\prime \prime \prime}(1) \leq 0 \Leftrightarrow \alpha \geq \frac{2 p b(p+1)[-p-2]}{6 b p(p+1)}=\frac{-(p+2)}{3}=\alpha_{1}
$$

Now, we can put

$$
f^{\prime \prime \prime}(t)=t^{p-3}\left(A t^{2}+B t+C\right) \Leftrightarrow f^{\prime \prime \prime}(t)=t^{p-3} f_{1}(t),
$$

with

$$
\begin{gathered}
A=\alpha b p(p+1)^{2}(p+2)-2 b p^{2}(p+2)(p+1) \\
B=2 \alpha b p(p+1)^{2}(p-1)+4(1-\alpha) b p(p+1)^{2}(p-1)-2 b p^{2}(p-1)(p+1)
\end{gathered}
$$

then, we obtain

$$
f_{1}^{\prime}(t)=2 A t+B=0 \Leftrightarrow t_{0}=\frac{-B}{2 A}>0
$$

We must have

$$
A<0 \text {, for } \alpha>\frac{2 b p^{2}(p+2)(p+1)}{b p(p+1)^{2}(p+2)}=\frac{2 p}{p+1}=\alpha_{2}, \text { with } p \in\left(-1, p_{1}\right)
$$

and

$$
B>0 \text {, for } \alpha<\frac{p+2}{p+1}=\alpha_{3} \text {, with } p \in\left(-1, p_{1}\right)
$$

such that

$$
t_{0}=\frac{-B}{2 A}<1<t \text {, for } \alpha>\frac{p+2}{3}=\alpha_{4} \text {, with } p \in\left(-1, p_{1}\right),
$$

so that $f_{1}$ is decreasing for $t>1$ and therefore, we obtain that $f^{\prime \prime \prime}(t)<0$ because $f^{\prime \prime \prime}(1) \leq 0$. By the same process we find that $f^{\prime \prime}(t)$ then that $f^{\prime}(t)$ and $f(t)$.

Finally in this part for $p \in\left(-1, p_{1}\right)$, we obtain that there exists $\alpha_{*}=\max \left(\alpha_{1}, \alpha_{2}, \alpha_{4}\right)$ and $\alpha_{3}$ such that for all $\alpha \in\left(\alpha_{*}, \alpha_{3}\right)$ we have:

$$
\alpha\left(\frac{a+b}{2}\right)+(1-\alpha)\left(\frac{2 a b}{a+b}\right) \leq \frac{p\left(a^{p+1}-b^{p+1}\right)}{(p+1)\left(a^{p}-b^{p}\right)} .
$$

To show the second inequality in this first case, we proceed by similar calculations. This is done by considering the function $g$ defined by

$$
\begin{aligned}
g(t)= & {[\beta b(p+1)-2 b p] t^{p+2}+[2 \beta b(p+1)+4(1-\beta) b(p+1)-2 b p] t^{p+1} } \\
& +[\beta b(p+1)] t^{p}+[-\beta b(p+1)] t^{2} \\
& +[-2 \beta b(p+1)-4(1-\beta) b(p+1)+2 b p] t+[-\beta b(p+1)+2 b p]
\end{aligned}
$$

So, after all the calculations, we get that for $p \in\left(-1, p_{1}\right)$, there exists 
$\alpha^{*}=\max \left(\beta_{1}, \beta_{2}, \beta_{3}, \beta_{4}\right)=\beta_{3}=\alpha_{3}=\frac{p+2}{p+1}$ such that $g(t) \geq 0$, for all $\beta>\alpha^{*}$. a.e:

$$
\frac{p\left(a^{p+1}-b^{p+1}\right)}{(p+1)\left(a^{p}-b^{p}\right)} \leq \beta\left(\frac{a+b}{2}\right)+(1-\beta)\left(\frac{2 a b}{a+b}\right)
$$

\section{Second case b):}

With similar calculations and by the same idea we obtain that for all $\alpha<0$ and $\beta \in\left(\frac{3}{2}, 2\right)$ then,

$$
\alpha\left(\frac{a+b}{2}\right)+(1-\alpha)\left(\frac{2 a b}{a+b}\right) \leq \frac{a-b}{\ln a-\ln b} \leq \beta\left(\frac{a+b}{2}\right)+(1-\beta)\left(\frac{2 a b}{a+b}\right) .
$$

\section{Third case c):}

By the method above and similar calculations, we also find that for all $\alpha<0$ and $\beta>\frac{1}{3}$ then,

$$
\alpha\left(\frac{a+b}{2}\right)+(1-\alpha)\left(\frac{2 a b}{a+b}\right) \leq \frac{a b(\ln a-\ln b)}{a-b} \leq \beta\left(\frac{a+b}{2}\right)+(1-\beta)\left(\frac{2 a b}{a+b}\right) .
$$

2) Assuming $a=b$.

We easily get:

$$
\begin{aligned}
& \alpha A(a, b)+(1-\alpha) H(a, b)=a=J_{p}(a, b) \\
& \beta A(a, b)+(1-\beta) H(a, b)=a=J_{p}(a, b),
\end{aligned}
$$

which shows that the double inequality holds for all of the parameters the $\alpha$ and $\beta$.

\section{Conclusions}

In our work, we studied the following double inequality

$$
\alpha A(a, b)+(1-\alpha) H(a, b) \leq J_{p}(a, b) \leq \beta A(a, b)+(1-\beta) H(a, b)
$$

by searching the best possible parameters such that (Inq) can be held.

\section{Firstly, we have inserted}

$$
f(t)=\alpha A(a, b)+(1-\alpha) H(a, b)-J_{p}(a, b)
$$

Without loss of generality, we have assumed that $a>b$ and let $t=\frac{a}{b}>1$ to determine the condition for $\alpha$ and $\beta$ to become $f(t) \leq 0$.

Secondly, have inserted

$$
g(t)=\beta A(a, b)+(1-\beta) H(a, b)-J_{p}(a, b)
$$

Without loss of generality, we assume that $a>b$ and let $t=\frac{a}{b}>1$ to de- 
termine the condition for $\alpha$ and $\beta$ to become $g(t) \geq 0$.

\section{Acknowledgements}

The authors gratefully acknowledge Qassim University, represented by the Deanship of Scientific Research, on the material support for this research WORK under the number (1061) during the academic year 1441AH/2020AD.

\section{Conflicts of Interest}

The authors declare no conflicts of interest regarding the publication of this paper.

\section{References}

[1] Bullen, P.S., Mitrinovic, D.S. and Vasic, M. (2013) Means and Their Inequalities, Vol. 31, Springer Science \& Business Media, Berlin.

[2] Ostasiewicz, S. and Ostasiewicz, W. (2000) Means and Their Applications. Annals of Operations Research, 97, 337-355. https://doi.org/10.1023/A:1018932425645

[3] Long, B.Y., Li, Y.M. and Chu, Y.M. (2012) Optimal Inequalities between Generalized Logarithmic, Identric and Power Means. International Journal of Pure and Applied Mathematics, 80, 41-51.

[4] Xia, W-F. and Chu, Y.-M. (2010) Optimal Inequalities Related to the Logarithmic, Identric, Arithmetic and Harmonic Means. Rev. Anal. Numer. Theor. Approx., 39, 176-183.

[5] Chen, J.-J., Lei, J.-J. and Long, B.-Y. (2017) Optimal Bounds for Neuman-Sandor Means in Term of the Convex Combination of the Logarithmic and the Second Seiffert Means. Journal of Inequalities and Applications, No. 1, 251. https://doi.org/10.1186/s13660-017-1516-7

[6] Newman, E. and Sandor, J. (2003) On the Schwab-Borcharrdt Means. Math. Pannon, 14, 253-266.

[7] Seiffert, S., Jungk, J.K.A. and Claassen, N. (1995) Observed and Calculated Potassium Uptake by Maize as Affected by Soil Water Content and Bulk Density. Agronomy Journal, 87, 1070-1077. https://doi.org/10.2134/agronj1995.00021962008700060007x

[8] Xu, H.-Z., Chu, Y.-M. and Qian, W.-M. (2018) Sharp Bounds for the Sandor-Yang Means in Terms of Arithmetic and Contra-Harmonic Means. Journal of Inequalities and Applications, No. 1, 127. https://doi.org/10.1186/s13660-018-1719-6

[9] Yang, Y.Y. and Qian, W.M. (2016) Two Optimal Inequalities Related to the Sandor-Yang Mean and One-Parameter Mean. Communications in Mathematical Research, 32, 352-358.

[10] Yang, Z.-H., Jiang, Y.-L., Song, Y.-Q. and Chu, Y.-M. (2014) Sharp Inequalities for Trigonometric Functions. In: Abstract and Applied Analysis, Vol. 2014. Hindawi. https://doi.org/10.1155/2014/601839

[11] Chu, Y.-M. and Hou, S.-W. (2012) Sharp Bounds for Seiffert Mean in Terms of Contraharmonic Mean. In: Abstract and Applied Analysis, Vol. 2012, Hindawi. https://doi.org/10.1186/1029-242X-2012-11

[12] Jiang, W.-D. and Qi, F. (2015) Sharp Bounds for the Neuman-Sandor Mean in Term of the Power and Contraharmonic Means. Cogent Mathematics, 2, 995951. https://doi.org/10.1080/23311835.2014.995951 